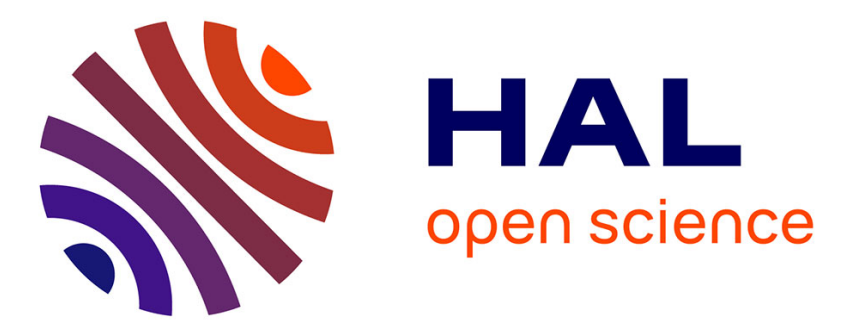

\title{
A WLS Estimator for Target Localization in a Cooperative Wireless Sensor Network
}

Slavisa Tomic, Marko Beko, Rui Dinis, Milan Tuba

\section{To cite this version:}

Slavisa Tomic, Marko Beko, Rui Dinis, Milan Tuba. A WLS Estimator for Target Localization in a Cooperative Wireless Sensor Network. 7th Doctoral Conference on Computing, Electrical and Industrial Systems (DoCEIS), Apr 2016, Costa de Caparica, Portugal. pp.273-283, 10.1007/978-3319-31165-4_27. hal-01438252

\section{HAL Id: hal-01438252 \\ https://hal.inria.fr/hal-01438252}

Submitted on 17 Jan 2017

HAL is a multi-disciplinary open access archive for the deposit and dissemination of scientific research documents, whether they are published or not. The documents may come from teaching and research institutions in France or abroad, or from public or private research centers.
L'archive ouverte pluridisciplinaire HAL, est destinée au dépôt et à la diffusion de documents scientifiques de niveau recherche, publiés ou non, émanant des établissements d'enseignement et de recherche français ou étrangers, des laboratoires publics ou privés.

\section{(c)(1)}

Distributed under a Creative Commons Attribution| 4.0 International License 


\title{
A WLS Estimator for Target Localization in a Cooperative Wireless Sensor Network
}

\author{
Slavisa Tomic ${ }^{4}$, Marko Beko ${ }^{1,3}$, Rui Dinis ${ }^{2,5}$, Milan Tuba ${ }^{6}$ \\ ${ }^{1}$ Universidade Lusófona de Humanidades e Tecnologias, Lisbon, Portugal \\ ${ }^{2}$ Instituto de Telecomunicações, Lisbon, Portugal \\ ${ }^{3}$ CTS, UNINOVA - Campus FCT/UNL, Caparica, Portugal \\ ${ }^{4}$ ISR/IST, LARSyS, Lisbon, Portugal \\ ${ }^{5}$ DEE/FCT/UNL, Caparica, Portugal \\ ${ }^{6}$ Megatrend University, Faculty of Computer Science, Belgrade, Serbia \\ \{s.tomic@ campus.fc.unl.pt, $\underline{\text { mbeko@ } @ \text { uninova.pt, }}$ rdinis@ fct.unl.pt, tuba@ieee.org
}

\begin{abstract}
This paper addresses target localization problem in a cooperative 3-D wireless sensor network (WSN). We employ non-traditional methodology which merges distance and angle measurements, respectively withdrawn from the received signal strength (RSS) and angle-of-arrival (AoA) information. Based on RSS measurement model and effortless geometry, a novel non-convex estimator according to the weighted least squares (WLS) criterion is obtained, which closely approximates the maximum likelihood (ML) estimator for small noise. It is shown that the devised estimator is appropriate for distributed implementation. Following the squared range (SR) approach, we propose a suboptimal SR-WLS estimator according to the generalized trust region sub-problem (GTRS) framework, to estimate the locations of all targets in the WSN. According to our simulations, the new estimator has excellent performance in a great variety of considered settings, in which the effectiveness of fusing two radio measurements is confirmed.
\end{abstract}

Keywords: Angle-of-arrival (AoA), generalized trust region sub-problem (GTRS), received signal strength (RSS), wireless sensor network (WSN).

\section{Introduction}

Accurate information about sensor's location is a key component in many practical applications. Wireless localization algorithms usually rely upon range measurements extracted from angle-of-arrival (AoA), received signal strength (RSS), time-of-arrival (ToA) information, or a combination of them [1].

In [2], a hybrid methodology which fuses distance and angle measures has been considered. Both estimators proposed in [2] deal with the non-cooperative target localization problem in a 3-D space: linear least squares (LS) and optimization based. The former one is a fairly simple estimator, and the later one uses Davidson-FletcherPowell algorithm [3]. The authors in [4] derived an LS and a maximum likelihood (ML) estimator for a hybrid scheme that fuses RSS difference (RSSD) and AoA measurements. The authors in [4] employed non-linear constrained optimization to estimate the unknown location from multiple RSS and AoA measurements. In [5], a 
selective weighted LS (WLS) estimator for mixed RSS/AoA localization problematic was presented. The target position was established by employing loaded distances from the two closest anchor measures, together with the serving base station AoA measurement. Another WLS estimator for a non-cooperative localization problem for the case where the transmitted power is not known was proposed in [6]. Nonetheless, similar as the method proposed in [5], the WLS method has been derived without requiring information about the statistical properties of RSS and AoA measurements, which might lead to significant performance degradation in practice. Also, the authors in [6] investigated a small-scale wireless sensor network (WSN) only, with extremely low noise power.

All of the above approaches examine non-collaborative localization problem only, where the location of a single target, which communicates with anchors exclusively, is established at a time. Contrarily to the above mentioned approaches, here, the target localization problem in an extensive WSN is considered, where the number of anchors is insufficient and the communication range of all sensors is limited (e.g., to prolong sensor's battery life). Hence, only some targets can directly communicate with anchors and sensor cooperation is required in order to acquire adequate amount of information to carry out the localization. Through employing the RSS spreading model and straightforward geometry, we first develop a new local non-convex estimator based on the WLS criterion that closely approximates the local ML one in the case where noise is low. Next, by following the squared range (SR) approach, we propose a suboptimal SR-WLS estimator based on the generalized trust region sub-problem (GTRS) framework, which is possible to solve exactly with a bisection method [7]. To the best of the authors' knowledge, distributed localization algorithms for hybrid RSS/AoA systems in cooperative WSNs are yet to be published.

\section{Relationship to Cyber-Physical Systems}

Cyber-physical systems (CPSs) have recently attracted much attention from both the educational and engineering public. These schemes are displaying an enormous potential in interacting with the physical world and creating its management more efficient. Integrating the computation and communication competencies into the modules of the physical surrounding [8] enables this. CPSs represent nowadays the new peer group of networks and embedded structures.

The placement of CPS raises numerous difficulties, where the most significant topic that has activated massive quantity of study is localization [8]. Basically, localization goals at estimating the location of CPS modules. In CPSs where data are closely related with the surroundings and the location at which they are produced, localization is a fundamental task.

Collaboration of a large number of scattered sensors in a WSN can be seen as a CPS. In such systems, localization is of a crucial importance, since a system may be configured to react locally to the variations within sensor records; hence, accurate determination of the location where the deviations arise is the key. Furthermore, services based on location-awareness represent a key component of countless wireless structures nowadays. By exploring the synergies between computational and physical 
components we can form smart environments which offer improved safety and efficiency in everyday life, e.g. smart parking, assistance for elderly or people with disabilities, monitoring of storage conditions and goods, etc.

\section{Problem Statement}

We examine a large-scale WSN with $N$ anchors and $M$ targets, that may also be viewed as a connected graph, $\mathcal{G}(\mathcal{V}, \mathcal{E})$, with $|\mathcal{V}|=M+N$ vertices and $|\mathcal{E}|$ edges (connections), where $|\cdot|$ represents the cardinality of a set. The set of targets and the set of anchors are respectively denoted as $\mathcal{T}(|\mathcal{T}|=M)$ and $\mathcal{A}(|\mathcal{A}|=N)$, and their locations are denoted by $\boldsymbol{x}_{1}, \boldsymbol{x}_{2}, \cdots, \boldsymbol{x}_{M}$ and $\boldsymbol{a}_{1}, \boldsymbol{a}_{2}, \cdots, \boldsymbol{a}_{N}, \quad\left(\boldsymbol{x}_{i}, \boldsymbol{a}_{j} \in \mathbb{R}^{3}, \forall i \in \mathcal{T}\right.$ and $\left.\forall j \in \mathcal{A}\right)$, respectively. Due to limited communication range, $R$, two sensors, $i$ and $j$, can interchange data if and only if they are inside the communication range of each other. The sets of all target/anchor and target/target edges are defined as $\varepsilon_{\mathcal{A}}=$ $\left\{(i, j):\left\|\boldsymbol{x}_{i}-\boldsymbol{a}_{j}\right\| \leq R, \forall i \in \mathcal{T}, \forall j \in \mathcal{A}\right\} \quad$ and $\quad \varepsilon_{\mathcal{T}}=\left\{(i, k):\left\|\boldsymbol{x}_{i}-\boldsymbol{x}_{k}\right\| \leq R, \forall i, k \in\right.$ $\mathcal{T}, i \neq k\}$, respectively.

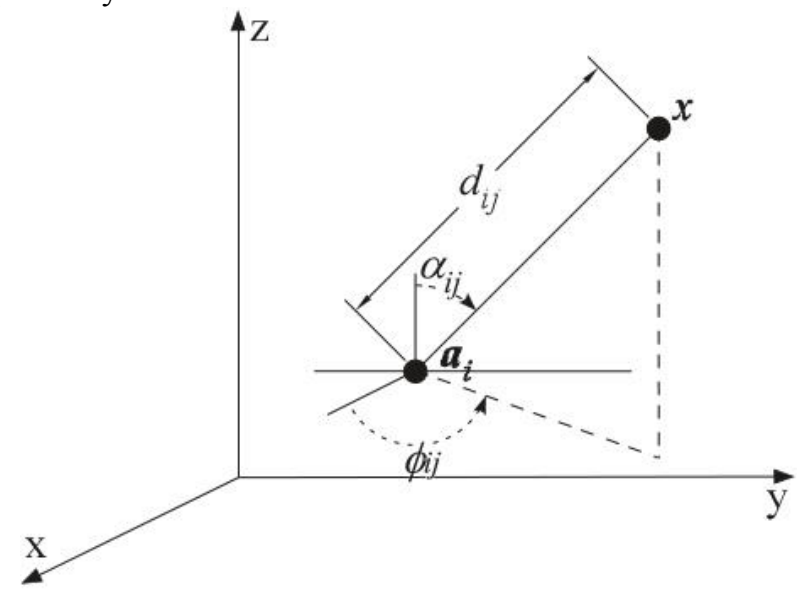

Fig. 1. Representation of a target and anchor locations within 3-D.

In favor of ease of expression, we describe a matrix $\boldsymbol{X}=\left[\boldsymbol{x}_{1}, \cdots, \boldsymbol{x}_{M}\right]\left(\boldsymbol{X} \in \mathbb{R}^{3 \times M}\right)$ as the matrix of all unknown target locations. These unknown locations are estimated with the means of a hybrid methodology which merges distance and angle measures.

Throughout this work, we imply that the distance measurements are extracted from the RSS measurements, because ranging based on RSS does not oblige additional hardware [1]. The path loss between two sensors is given by:

$$
L_{i j}=L_{0}+10 \gamma \log _{10} \frac{\left\|\boldsymbol{x}_{i}-\widehat{\boldsymbol{a}}_{j}\right\|}{d_{0}}+n_{i j}, \forall(i, j) \in \varepsilon_{\mathcal{A}} \cup \varepsilon_{\mathcal{T}}
$$

(see [9],[10]) where $L_{0}$ represents the path loss value at a reference distance $d_{0}$ $\left(\left\|\boldsymbol{x}_{i}-\widehat{\boldsymbol{a}}_{j}\right\| \geq d_{0}\right), \gamma$ is the path loss exponent (PLE), $\widehat{\boldsymbol{a}}_{j}$ is the $j$-th neighbor of the $i$-th target $\left(\boldsymbol{a}_{j}\right.$ if $j \in \mathcal{A}, \boldsymbol{x}_{j}$ if $\left.j \in \mathcal{T}\right)$, and $n_{i j}$ is the log-normal shadowing considered as a 
Gaussian random variable, i.e., $n_{i j} \sim \mathcal{N}\left(0, \sigma_{n_{i j}}^{2}\right)$. The target/target path loss measures are assumed to be symmetric ${ }^{1}$.

Fig. 1 gives a representation of a target and anchor locations within 3-D. Looking at Fig. 1, $\boldsymbol{x}_{i}=\left[x_{i 1}, x_{i 2}, x_{i 3}\right]^{T}$ and $\boldsymbol{a}_{j}=\left[a_{j 1}, a_{j 2}, a_{j 3}\right]^{T}$ represent the coordinates of the $i$ th target (not known) and the coordinates of the $j$-th anchor (known) respectively, whereas $d_{i j}, \phi_{i j}$ and $\alpha_{i j}$ respectively stand for the range, azimuth and elevation angle amongst the $i$-th target and the $j$-th anchor. According to the measurement model (1) the ML distance between two sensors is obtained as follows [1]:

$$
\hat{d}_{i j}=d_{0} 10^{\frac{L_{i j}-L_{0}}{10 \gamma}}, \forall(i, j) \in \varepsilon_{\mathcal{A}} \cup \varepsilon_{\mathcal{T}} .
$$

By applying simple geometry, azimuth and elevation angle measurements can be modeled respectively as [2]:

$$
\phi_{i j}=\tan ^{-1}\left(\frac{x_{i 2}-a_{j 2}}{x_{i 1}-a_{j 1}}\right)+m_{i j}, \forall(i, j) \in \varepsilon_{\mathcal{A}},
$$

and

$$
\alpha_{i j}=\cos ^{-1}\left(\frac{x_{i 3}-a_{j 3}}{\left\|x_{i}-a_{j}\right\|}\right)+v_{i j}, \forall(i, j) \in \varepsilon_{\mathcal{A}},
$$

According to (1), (3) and (4), we can obtain the ML estimator as [11]:

$$
\widehat{\boldsymbol{X}}=\underset{\boldsymbol{X}}{\arg \min } \sum_{i=1}^{3\left|\varepsilon_{\mathcal{A}}\right|+\left|\varepsilon_{\mathcal{T}}\right|} \frac{1}{\sigma_{i}^{2}}\left[\theta_{i}-f_{i}(\boldsymbol{X})\right]^{2},
$$

where $\boldsymbol{\theta}=\left[\boldsymbol{L}^{T}, \boldsymbol{\phi}^{T}, \boldsymbol{\alpha}^{T}\right]^{T}\left(\boldsymbol{\theta} \in \mathbb{R}^{3\left|\varepsilon_{\mathcal{A}}\right|+\left|\varepsilon_{\mathcal{T}}\right|}\right)$, with $\boldsymbol{L}=\left[L_{i j}\right]^{T}, \forall(i, j) \in \varepsilon_{\mathcal{A}} \cup \varepsilon_{\mathcal{T}}, \boldsymbol{\phi}=$ $\left[\phi_{i j}\right]^{T}, \forall(i, j) \in \varepsilon_{\mathcal{A}}, \boldsymbol{\alpha}=\left[\alpha_{i j}\right]^{T}, \forall(i, j) \in \varepsilon_{\mathcal{A}}$, and

$$
\boldsymbol{f}(\boldsymbol{X})=\left[\begin{array}{c}
\vdots \\
L_{0}+10 \gamma \log _{10} \frac{\left\|\boldsymbol{x}_{i}-\widehat{\boldsymbol{a}}_{j}\right\|}{d_{0}} \\
\vdots \\
\tan ^{-1}\left(\frac{x_{i 2}-a_{j 2}}{x_{i 1}-a_{j 1}}\right) \\
\vdots \\
\cos ^{-1}\left(\frac{x_{i 3}-a_{j 3}}{\left\|\boldsymbol{x}_{i}-\boldsymbol{a}_{j}\right\|}\right) \\
\vdots
\end{array}\right], \boldsymbol{\sigma}=\left[\begin{array}{c}
\vdots \\
\sigma_{n_{i j}} \\
\vdots \\
\sigma_{m_{i j}} \\
\vdots \\
\sigma_{v_{i j}} \\
\vdots
\end{array}\right] .
$$

The non-convex LS estimator in (5) does not have a closed-form solution. Nonetheless, we will show that it can be transformed into GTRS framework and solved efficiently.

${ }^{1}$ This assumption is made without loss of generality; it is readily seen that, if $L_{i j}^{T} \neq L_{j i}^{T}, \forall(i, j) \in$ $\mathcal{E}_{\mathcal{T}}$, then it is enough to replace $L_{i j}^{T} \leftarrow\left(L_{i j}^{T}+L_{j i}^{T}\right) / 2$ and $L_{j i}^{T} \leftarrow\left(L_{i j}^{T}+L_{j i}^{T}\right) / 2$ when solving the localization problem. 


\section{The Proposed SR-WLS Estimator}

Assuming that the initial location estimations of the targets, $\widehat{\boldsymbol{X}}^{(0)}$, are given, the ML in (5) can be solved locally per target, resorting merely to the data collected from its neighbors and employing an iterative methodology. Consequently, target $i$ updates its location estimation in each iteration, $t$, by solving the below local ML estimator:

$$
\widehat{\boldsymbol{x}}_{i}^{(t+1)}=\underset{\boldsymbol{x}_{\boldsymbol{i}}}{\arg \min } \sum_{j=1}^{3\left|\varepsilon_{\mathcal{A}_{i}}\right|+\left|\varepsilon_{\mathcal{T}_{i}}\right|} \frac{1}{\sigma_{j}^{2}}\left[\theta_{j}-f_{j}\left(\boldsymbol{x}_{i}\right)\right]^{2}, \forall i \in \mathcal{T},
$$

where $\varepsilon_{A_{i}}=\left\{j:(i, j) \in \varepsilon_{A}\right\}$ and $\varepsilon_{T_{i}}=\left\{k:(i, k) \in \varepsilon_{T}\right\}$ represent the set of all anchor and all target neighbors of the target $i$ respectively.

Given $\widehat{\boldsymbol{X}}^{(0)}$, for the case where noise power is low, out of (1) we can write:

$$
\lambda_{i j}\left\|\boldsymbol{x}_{i}-\widehat{\boldsymbol{a}}_{j}\right\|^{2} \approx d_{0}^{2}, \forall i \in \mathcal{T}, \forall j \in \varepsilon_{\mathcal{A}_{i}} \cup \varepsilon_{\mathcal{T}_{i}},
$$

where $\lambda_{i j}=10^{\frac{L_{0}-L_{i j}}{5 \gamma}}$. Similarly, from (3) and (4) we respectively get:

$$
\boldsymbol{c}_{i j}^{T}\left(\boldsymbol{x}_{i}-\boldsymbol{a}_{j}\right) \approx 0, \forall i \in \mathcal{T}, \forall j \in \varepsilon_{\mathcal{A}_{i}},
$$

and

$$
\boldsymbol{k}^{T}\left(\boldsymbol{x}_{i}-\boldsymbol{a}_{j}\right) \approx\left\|\boldsymbol{x}_{i}-\boldsymbol{a}_{j}\right\| \cos \left(\alpha_{i j}\right), \forall i \in \mathcal{T}, \forall j \in \varepsilon_{\mathcal{A}_{i}} .
$$

where $c_{i j}=\left[-\sin \left(\phi_{i j}\right), \cos \left(\phi_{i j}\right), 0\right]^{T}$ and $\boldsymbol{k}=[0,0,1]^{T}$. To grant more significance to nearby connections, bring into play weights, $\boldsymbol{w}=\left[\sqrt{w_{i j}}\right]$, where $w_{i j}=1-$ $\hat{d}_{i j}$

$$
/ \sum_{\forall i \in \mathcal{T}, \forall j \in \varepsilon_{\mathcal{A}_{i}} \cup \varepsilon_{\mathcal{T}_{i}}} \hat{d}_{i j} \text {, and substitute }\left\|\boldsymbol{x}_{i}-\widehat{\boldsymbol{a}}_{j}\right\| \text { in (9) with } \hat{d}_{i j} \text { described in }
$$

According to the WLS criterion and (7), (8) and (9) every target updates its location through minimization of the below estimator:

$$
\begin{gathered}
\widehat{\boldsymbol{x}}_{i}^{(t+1)}=\underset{\boldsymbol{x}_{\boldsymbol{i}}}{\arg \min } \sum_{j \in \mathcal{E}_{\mathcal{A}_{i}} \cup \mathcal{E}_{\mathcal{T}_{i}}} w_{i j}\left(\lambda_{i j}\left\|\boldsymbol{x}_{i}-\widehat{\boldsymbol{a}}_{j}\right\|^{2}-d_{0}^{2}\right)^{2}+ \\
\sum_{j \in \varepsilon_{\mathcal{A}_{i}}} w_{i j}\left(\boldsymbol{c}_{i j}^{T}\left(\boldsymbol{x}_{i}-\boldsymbol{a}_{j}\right)\right)^{2}+\sum_{j \in \varepsilon_{\mathcal{A}_{i}}} w_{i j}\left(\boldsymbol{k}^{T}\left(\boldsymbol{x}_{i}-\boldsymbol{a}_{j}\right)-\hat{d}_{i j} \cos \left(\alpha_{i j}^{\mathcal{A}}\right)\right)^{2} .
\end{gathered}
$$

The above WLS problem is not convex and does not have a closed-form solution. Still, (10) may be expressed as a quadratic programming problem of which the global solution is found effortlessly [7]. Applying the replacement $\boldsymbol{y}_{i}=\left[\boldsymbol{x}_{\boldsymbol{i}}^{\boldsymbol{T}},\left\|\boldsymbol{x}_{\boldsymbol{i}}\right\|^{2}\right]^{T}, \forall i \in \mathcal{T}$, equation (10) is modified as:

subject to

$$
\boldsymbol{y}_{i}^{(t+1)}=\underset{\boldsymbol{y}_{i}}{\arg \min }\left\|\boldsymbol{W}\left(\boldsymbol{A} \boldsymbol{y}_{i}-\boldsymbol{b}\right)\right\|^{2}
$$

$$
\boldsymbol{y}_{i}^{T} \mathbf{D} \mathbf{y}_{\mathrm{i}}+2 l^{T} \boldsymbol{y}_{i}=0
$$

where $\boldsymbol{W}=\boldsymbol{I}_{\mathbf{3}} \otimes \operatorname{diag}(\boldsymbol{w})$, and $\otimes$ denotes the Kronecker product, $\boldsymbol{I}_{q}$ represents the $q$ dimensional identity matrix, and 
$\boldsymbol{A}=\left[\begin{array}{cc}\vdots & \vdots \\ -2 \lambda_{i j} \widehat{\boldsymbol{a}}_{j}^{T} & \lambda_{i j} \\ \vdots & \vdots \\ \boldsymbol{c}_{i j}^{T} & 0 \\ \vdots & \vdots \\ \boldsymbol{k}^{T} & 0 \\ \vdots & \vdots\end{array}\right], \boldsymbol{b}=\left[\begin{array}{c}\vdots \\ d_{0}^{2}-\lambda_{i j}\left\|\widehat{\boldsymbol{a}}_{j}\right\|^{2} \\ \vdots \\ \boldsymbol{c}_{i j}^{T} \boldsymbol{a}_{j} \\ \vdots \\ \boldsymbol{k}^{T} \boldsymbol{a}_{j}+\hat{d}_{i j} \cos \left(\alpha_{i j}\right) \\ \vdots\end{array}\right], \boldsymbol{D}=\left[\begin{array}{cc}\boldsymbol{I}_{3} & \mathbf{0}_{3 \times 1} \\ \mathbf{0}_{1 \times 3} & 0\end{array}\right], \boldsymbol{l}=\left[\begin{array}{c}\mathbf{0}_{3 \times 1} \\ \frac{1}{2}\end{array}\right]$,

i.e., $\boldsymbol{A} \in \mathbb{R}^{3\left|\varepsilon_{\mathcal{A}_{i}}\right|+\left|\varepsilon_{\mathcal{J}_{i}}\right| \times 4}, \boldsymbol{b} \in \mathbb{R}^{3\left|\varepsilon_{\mathcal{A}_{i}}\right|+\left|\varepsilon_{\mathcal{J}_{i}}\right| \times 1}, \boldsymbol{W} \in \mathbb{R}^{3\left|\varepsilon_{\mathcal{A}_{i}}\right|+\left|\varepsilon_{\mathcal{J}_{i}}\right| \times 3\left|\varepsilon_{\mathcal{A}_{i}}\right|+\left|\varepsilon_{\mathcal{J}_{i}}\right|}$, and $\mathbf{0}_{p \times q}$ is the $p \times q$-dimensional matrix of all zeros.

Both the objective and the restraint functions in (11) are quadratic. This type of problem is identified as GTRS [7]; it may be solved exactly by a bisection method [7].

Assuming that $\mathcal{C}$ is the set of colors of the nodes (in order to coordinate the network [12]), Algorithm 1 encapsulates the suggested distributed SR-WLS algorithm. Lines 5-7 are carried out concurrently by all targets $i \in \mathcal{C}_{c}$, which might reduce the realization time of the approach. Information interchange occurs exclusively at Line 7, when targets send their location updates $\widehat{\boldsymbol{x}}_{\boldsymbol{i}}^{(t+1)}$ to their neighbors. Because $\widehat{\boldsymbol{x}}_{\boldsymbol{i}}^{(t+1)} \in \mathbb{R}^{3}$, one concludes that the new approach obliges a transmission of a maximum $3 \times T_{\max } \times M$ real values. The worst case computational complexity of the proposed method is linear, i.e., $T_{\max } \times M \times \mathcal{O}\left(N_{\max } \times \max _{i}\left\{3\left|\varepsilon_{\mathcal{A}_{i}}\right|+\left|\varepsilon_{\mathcal{T}_{i}}\right|\right\}\right)$, where $N_{\text {max }}$ denotes the maximum allowed number of iterations of the bisection method. In the further text, we will refer to Algorithm 1 as "SR-WLS".

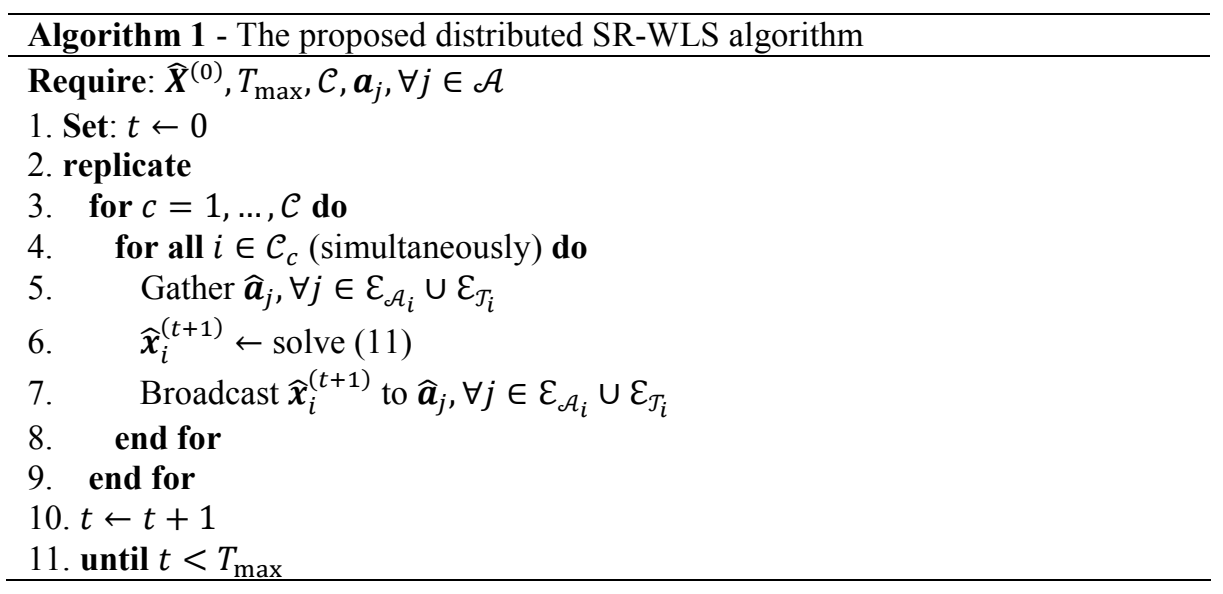

\section{Performance Evaluation}

A set of simulation results is presented here with the intention of assessment of the performance of the new approach in the view of the estimation precision and convergence. All of the considered approaches were solved via MATLAB. In order to demonstrate the benefit of fusing two radio measurements versus traditional 
localization systems, we include also the performance results of the proposed method when only RSS measurements are employed, called here "SR-WLS RSS".

In order to produce the radio measures, models (1), (3) and (4) are employed. A random arrangement of sensors within a box with the edge span $B=20 \mathrm{~m}$ in each Monte Carlo $\left(M_{c}\right)$ trial was considered. If not declared otherwise, the PLE was set to $\gamma=3$, the reference distance to $d_{0}=1 \mathrm{~m}$, the reference path loss to $L_{0}=40 \mathrm{~dB}$, and the maximum number of steps in the bisection procedure was $N_{\max }=30$. In practice however, it is almost impossible to perfectly estimate the value of the PLE. Thus, in order to account for a realistic measurement model mismatch and test the robustness of the considered approaches to flawed knowledge of the PLE, the true PLE for each connection was chosen from a uniform distribution on an interval $\gamma_{i j} \in$ $[2.7,3.3], \forall(i, j) \in \varepsilon_{\mathcal{A}} \cup \varepsilon_{\mathcal{T}}$. Finally, the zero estimate of the targets' locations, $\widehat{\boldsymbol{X}}^{(0)}$, was assumed to be at the intersection of the big diagonals of the cube area. The main performance criterion used is the normalized root mean square error (NRMSE), defined as

$$
\text { NRMSE }=\sqrt{\sum_{i=1}^{M_{c}} \sum_{j=1}^{M} \frac{\left\|x_{i j}-\widehat{x}_{i j}\right\|}{M M_{c}}}
$$

where $\widehat{\boldsymbol{x}}_{i j}$ represents the estimation of the real location of the $j$-th target, $\boldsymbol{x}_{i j}$, in the $i$-th $M_{c}$ trial.

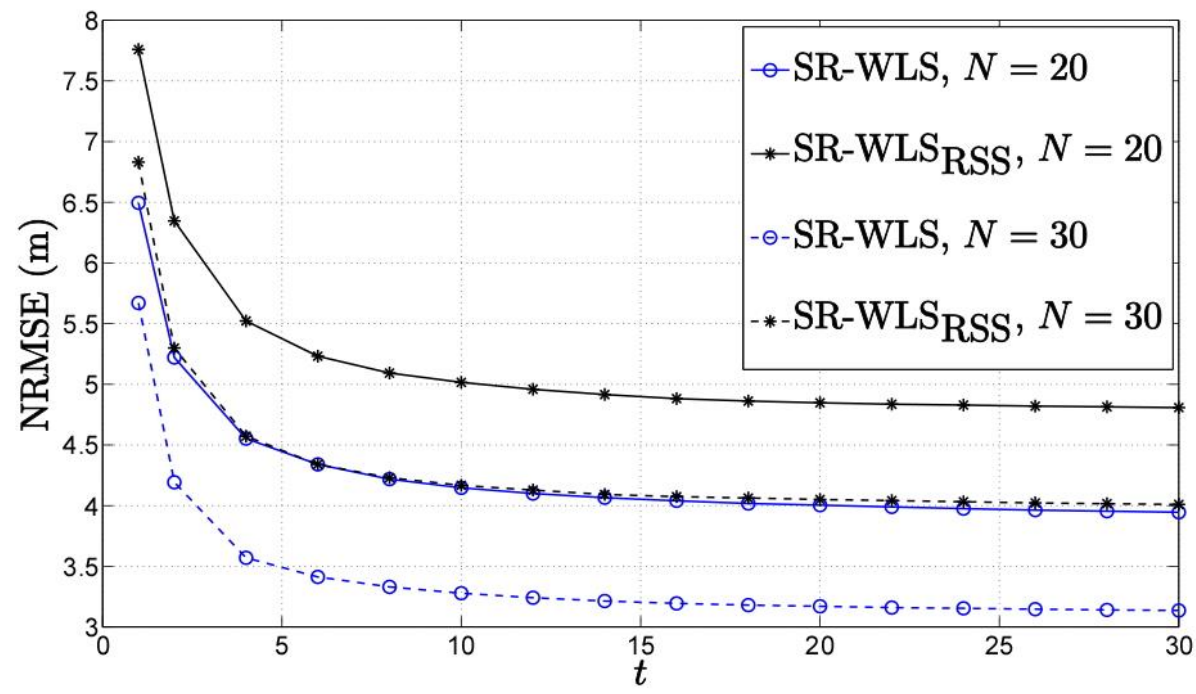

Fig. 2. NRMSE set against $t$ assessment, when $M=50, R=6.5 \mathrm{~m}, \boldsymbol{\sigma}_{\boldsymbol{n}_{i j}}=\mathbf{3} \mathrm{dB}, \boldsymbol{\sigma}_{\boldsymbol{m}_{i j}}=$ $6 \mathrm{deg}, \sigma_{v_{i j}}=6 \mathrm{deg}, \gamma_{i j} \in[2.7,3.3], \gamma=3, \mathrm{~B}=20 \mathrm{~m}, L_{0}=40 \mathrm{~dB}, d_{0}=1 \mathrm{~m}, M_{c}=500$.

Figs. 2 and 3 illustrate the NRMSE versus $t$ performance when $R=6.5 \mathrm{~m}, \sigma_{n_{i j}}=$ $3 \mathrm{~dB}, \sigma_{m_{i j}}=6 \mathrm{deg}$, and $\sigma_{v_{i j}}=6 \mathrm{deg}$, for $M=50, N=20$ and $N=30$, and $N=20$, $M=50$ and $M=60$, respectively. From these figures, we can notice that the output from considered approaches improves as $t$ grows, as anticipated. Also, it can be seen from Fig. 2 that the performance of all algorithms improves considerably as more 
anchors are included into the network. This behavior is anticipated, because when $N$ is increased more reliable information is introduced in the network. Furthermore, Fig. 3 reveals that both methods need a somewhat elevated number of repetitions to converge for augmented $M$. Nonetheless, the estimation precision of the considered algorithms improves when more targets are added in the network. Moreover, from Figs. 2 and 3 one can perceive that all major changes in the performance for the considered algorithms occur in the first few iterations $(t \leq 15)$, and that the performance gain is insignificant afterwards. This result is very important because it shows that our approach necessitates a low number of signal broadcast, which might augment the exploitation productivity of the spectrum, a valuable resource for wireless communications. It also shows that our algorithm is energy efficient; the communication stage is considerably more demanding (in terms of energy) than the data processing one [1],[12].

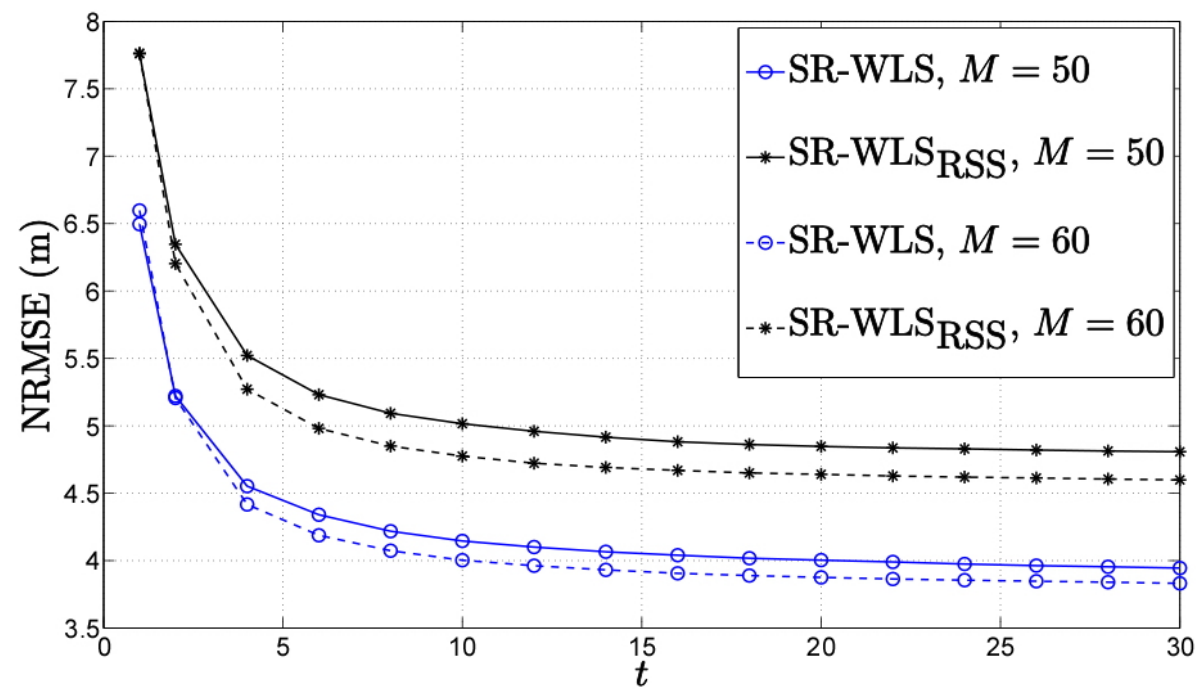

Fig. 3. NRMSE versus $t$ comparison, when $N=20, R=6.5 \mathrm{~m}, \sigma_{n_{i j}}=3 \mathrm{~dB}, \sigma_{m_{i j}}=$ $6 \mathrm{deg}, \sigma_{v_{i j}}=6 \mathrm{deg}, \gamma_{i j} \in[2.7,3.3], \gamma=3, \mathrm{~B}=20 \mathrm{~m}, L_{0}=40 \mathrm{~dB}, d_{0}=1 \mathrm{~m}, M_{c}=500$.

Fig. 4 illustrates the NRMSE versus $\sigma_{n_{i j}}(\mathrm{~dB})$ assessment, when $N=20, M=$ $50, R=6.5 \mathrm{~m}$, and $T_{\max }=30$. In this figure, one can perceive the performance degradation of the proposed algorithm as the quality of the RSS measurement reduces, as projected. Nonetheless, we can see from the figure that the deterioration in the performance is lower than $15 \%$ for the proposed algorithm, which is relatively low for the considered noise range. Finally, Fig. 4 confirms the effectiveness of measurement fusion, showing a gain of almost $1 \mathrm{~m}$ when the hybrid system is employed in comparison with the traditional RSS system. 


\section{Conclusion}

Here, the hybrid RSS/AoA target localization problem in a collaborative 3-D WSN was addressed. By fusing information from RSS and AoA, we devised a new problem formulation based on a nonconvex WLS. We showed that the derived novel estimator is suitable for distributed implementation, and we presented a novel non-centralized procedure founded on the GTRS framework. This new algorithm is light in terms of computational complexity, and it can provide accurate localization in a variety of scenarios, having exhibited excellent performance both in view of the estimation precision and convergence. Moreover, the simulation results confirmed the effectiveness of combining RSS and AoA radio measurements in comparison with conventional RSS localization system, displaying a notable enhancement of the estimation precision.

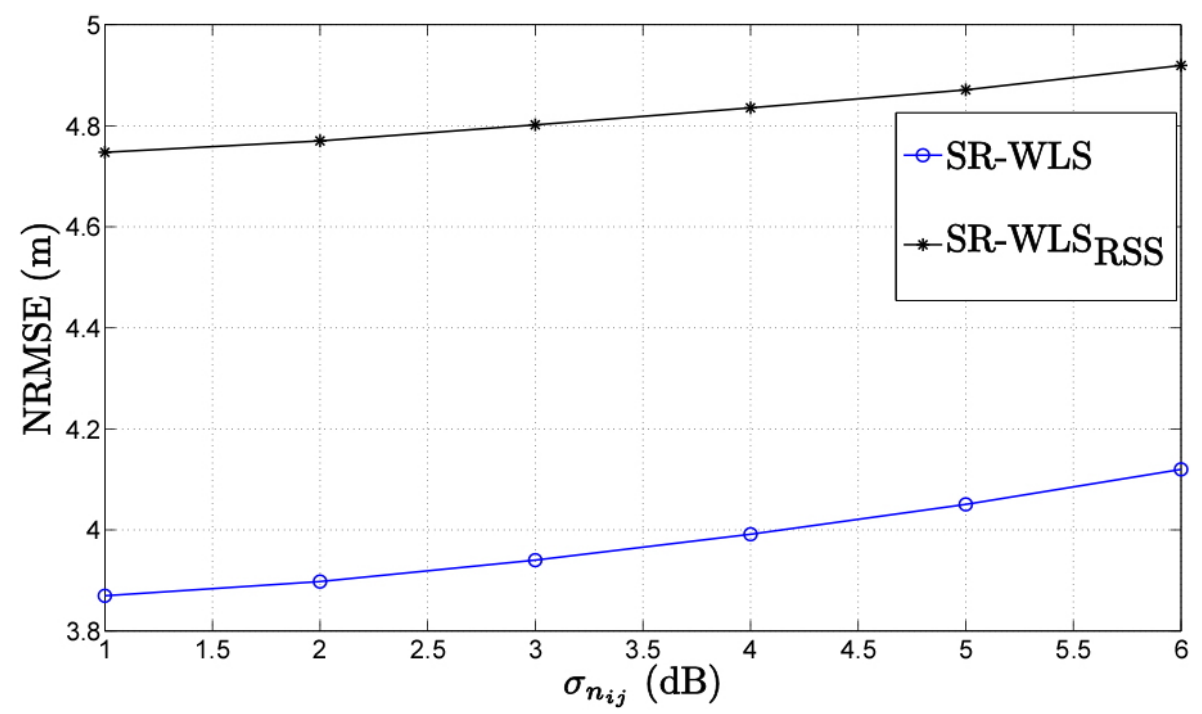

Fig. 4. NRMSE versus $\sigma_{n_{i j}}(\mathrm{~dB})$ assessment, for $N=20, M=50, R=6.5 \mathrm{~m}, \sigma_{m_{i j}}=$ $1 \mathrm{deg}, \sigma_{v_{i j}}=1 \mathrm{deg}, \gamma_{i j} \in[2.7,3.3], \gamma=3, T_{\max }=30, B=20 \mathrm{~m}, L_{0}=40 \mathrm{~dB}, d_{0}=1 \mathrm{~m}, M_{c}=$ 500 .

Acknowledgments. This work was partially supported by Fundação para a Ciência e a Tecnologia under Projects PEst-OE/EEI/UI0066/2014, and PEst-OE/EEI/LA0008/ 2013 (IT pluriannual founding and HETNET), PEst-OE/EEI/UI0066/2011 (UNINOVA pluriannual founding), ADIN PTDC/EEI-TEL/2990/2012, COPWIN PTDC/EEI-TEL/1417/2012 and PTDC/EEITEL/6308/2014-HAMLeT, as well as the grants SFRH/BPD/108232/2015, SFRH/BD/91126/2012 and Ciência 2008 PostDoctoral Research grant. 


\section{References}

1. N. Patwari. Location Estimation in Sensor Networks. Ph.D. Thesis, University of Michigan, Ann Arbor, MI, USA, 2005.

2. K. Yu, "3-D Localization Error Analysis in Wireless Networks," IEEE Trans. Wirel. Commun., vol. 6, no. 10, pp. 3473--3481, Oct. 2007.

3. R. Fletcher. Practical Methods of Optimization. John Wiley \& Sons, Chichester, UK, 1987.

4. S. Wang, B. R. Jackson, and R. Inkol, "Hybrid RSS/AOA Emitter Location Estimation Based on Least Squares and Maximum Likelihood Criteria," IEEE QBSC, pp. 24--29, Jun. 2012.

5. L. Gazzah, L. Najjar, and H. Besbes, "Selective Hybrid RSS/AOA Weighting Algorithm for NLOS Intra Cell Localization," IEEE WCNC, pp. 2546--2551, Apr. 2014.

6. Y. T. Chan, F. Chan, W. Read, B. R. Jackson, and B. H. Lee, "Hybrid Localization of an Emitter by Combining Angle-of-Arrival and Received Signal Strength Measurements," IEEE CCECE, pp. 1--5, May. 2014

7. A. Beck, P. Stoica, and J. Li, "Exact and Approximate Solutions of Source Localization Problems," IEEE Trans. Sig. Process., vol. 56, no. 5, pp. 1770--1778, May 2008.

8. A. Koubaa and M. B. Jamaa, "Taxonomy of Fundamental Concepts of Localization in CyberPhysical and Sensor Networks," Technical Report, CISTER, Research Center in Real-Time \& Embedded Computing Systems, Feb. 2013.

9. T. S. Rappaport. Wireless Communications: Principles and Practice. Prentice-Hall: Upper Saddle River, NJ, USA, 1996.

10. M. L. Sichitiu and V. Ramadurai, "Localization of Wireless Sensor Networks with a Mobile Beacon," IEEE MASS, pp. 174--183, Oct. 2004.

11. S. M. Kay. Fundamentals of Statistical Signal Processing: Estimation Theory. Prentice-Hall: Upper Saddle River, NJ, USA, 1993.

12. S. Tomic, M. Beko, and R. Dinis, "Distributed RSS-Based Localization in Wireless Sensor Networks Based on Second-Order Cone Programming," Sensors, vol. 14, no. 10, pp. 18410-18432 , Oct. 2014 